\title{
Comparison of Two Analytical Methods for Numerical Hypersensitivity of Vibration Modes with Close Frequencies
}

\author{
X.L. Liu \\ Laboratório Nacional de Engenharia Civil, Av. do Brasil, 101, 1700-066 Lisboa, Portugal
}

\section{C.S. Oliveira}

Departamento de Engenharia Civil e Arquitectura, Instituto Superior Técnico,

Av. Rovisco Pais, 1049-001 Lisboa, Portugal

(Received 22 February 2001; revised 29 January 2002; accepted 21 February 2002)

\begin{abstract}
This paper provides a comparison of numerical approaches, in terms of qualitative and quantitative analyses, to study the hypersensitivity of modes with close frequencies. The existence of close frequencies and strong modal couplings can cause rapid changes under a small variation of parameters, observed as the curve veering of frequencies and the mode localisation. Perturbation analysis with a discrete frequency is used for qualitative analysis, and shows a tendency of veering and localisation of large but miscalculated modal changes. On the other hand, quantitative analysis, which considers the specific characteristics of the modes of close frequencies, can give a more precise measurement. Three examples are given, which show the numerical differences between qualitative and quantitative analyses.
\end{abstract}

\section{INTRODUCTION}

Modal analysis, which can be conducted theoretically or through experimental testing, plays an important role in understanding the behaviour of a vibrating system. Changes of frequencies and mode shapes following the variation of parameters are of interest to researchers and engineers, such as in sensitivity analysis and updating of system designs. JeanLouis Guyader ${ }^{1}$ has brought the topic "hypersensitivity" to the attention of researchers and engineers in his editorial "Sensitivity of Structural Vibration to Structural Uncertancies". In this editorial he states that "this challenging problem needs to be addressed in structural and acoustics research programmes in the next decade" and "special attention should be paid to progress in this field of research".

Mode localisation and frequency curve veering are phenomena, which display rapid modal changes in vibrating systems. Early studies ${ }^{2-8}$ demonstrated that mode shapes can undergo dramatic changes within a small part of a structure. Nonlinear localised modes were found also in periodic oscillators ${ }^{9,10}$ and continuous systems ${ }^{11,12}$. Experiments on disordered multi-span beams ${ }^{13,14}$ and on a ribbed antenna ${ }^{15}$ proved the occurrence of mode localisation. Frequency curve veering or loci veering have been observed in various vibrating systems, such as rectangular membranes ${ }^{16}$, orthotropic rectangular plates ${ }^{17}$, cables and chains ${ }^{18}$, rotating circular strings ${ }^{19}$, coupled oscillators ${ }^{20}$, multi-span beams ${ }^{13}$, and weakly coupled pendulums ${ }^{5,21}$.

Mode localisation has been used in vibration control. ${ }^{22}$ Other controlling techniques include the use of damping ${ }^{23}$ and actuators ${ }^{24}$.

Pierre $^{21}$ concluded that when some type of perturbation is introduced into nearly periodic structures with close modal frequencies; strong mode localisation and frequency curve veering are manifestations of the same drastic phenomena. Following a small perturbation such as a minor geometrical modification in a vibrating system, these phenomena are indeed seen to be a case of hypersensitivity.

Although the occurrence of curve veering and mode localisation in vibrating systems with close modal frequencies has been studied by many researches, there are only a few references on the numerical analysis needed to predict them. ${ }^{5,13,20,21,25-31}$ The perturbation method, as an approximate and efficient analytical approach, has been used to determine these phenomena. The conventional perturbation algorithm ${ }^{25,26}$, which is only applicable to a single mode, cannot be used to produce a correct prediction for curve veering and mode localisation. This method is incorrect because of the introduction of two close frequencies in the perturbation formulation. Consequently, an advanced perturbation algorithm, in terms of the quantitative analysis for a precise measurement, should be used.

In this paper, analytical methods, in terms of qualitative and quantitative analyses, to predict and measure the rapid modal changes in the curve veering and mode localisation with close modal frequencies, are examined. In the following section, weakly coupled pendulums are presented as an illustration for the case of close modal frequencies and the occurrence of the phenomena. Later, qualitative and quantitative methods are reviewed. The first method uses the perturbation formulas for a single mode with a discrete frequency. The curve veering or mode localisation is observed from this analysis when there is a large value obtained from the incorrect prediction. On the other hand, accurate analysis is obtained from the second method, which uses formulas for the close modal frequencies. The modal changes obtained with these two methods will be demonstrated in three examples following. 